\title{
Effects of Ropivacaine Combined with Ketorolac Transverse Abdominis Plane Block on Postoperative Analgesia in Patients Undergoing Gynecological Laparoscopic Surgery
}

\author{
Yidong Hao, Rui Xia*, Qifei Li, Xiaoyong Zhao, Xiangyu Liu \\ The First Affiliated Clinical Medical College of Yangtze University, Jingzhou 434023, Hubei, China \\ Orcid of the first author: 0000-0002-2336-6967 \\ Email: xiarui318@163.com
}

\begin{abstract}
Objective - To observe the effects of ropivacaine combined with ketorolac transverse abdominis plane (TAP) block on postoperative analgesia in patients undergoing gynecological laparoscopic surgery. Methods - 90 ASA I or I patients, aged 18-65 years and weighting 40-80 kg, scheduled for gynecological laparoscopic surgery were recruited and divided into three groups using random number table:ropivacaine combined with ketorolac $15 \mathrm{mg}$ /side for TAP block (KR group), ropivacaine for TAP block and $30 \mathrm{mg}$ preoperatively intravenous ketorolac (KIVR group) and ropivacaine for TAP block alone (R group) with 30 patients in each group. Intraoperative opioid consumption, Bruggrmann comfort scale (BCS) scores at $2 \mathrm{~h}\left(\mathrm{~T}_{1}\right), 4 \mathrm{~h}\left(\mathrm{~T}_{2}\right), 8 \mathrm{~h}\left(\mathrm{~T}_{3}\right), 12 \mathrm{~h}\left(\mathrm{~T}_{4}\right), 16 \mathrm{~h}\left(\mathrm{~T}_{5}\right), 24 \mathrm{~h}\left(\mathrm{~T}_{6}\right)$ after surgery were recorded. Adverse reactions such as nausea and vomiting, respiratory depression, local anesthetic poisoning, gastrointestinal bleeding, and complications related to TAP block operation were recorded. Results - The total consumpsion of sufentanil was higher in the R group than that in the KIVR group and KR group $(\mathrm{P}<0.05)$. The total dosage of remifentanil in $\mathrm{R}$ group was higher than that in $\mathrm{KR}$ group $(P<$ 0.05). The BCS scores of the three groups at $\mathrm{T}_{3}, \mathrm{~T}_{4}, \mathrm{~T}_{5}$ and $\mathrm{T}_{6}$ were lower in $\mathrm{R}$ group than that in $\mathrm{KR}$ group $(P<0.05)$ while $\mathrm{R}$ Group was lower than KIVR group at $\mathrm{T}_{3}$ and $\mathrm{T}_{4}(P<0.05)$, and KIVR group was lower than KR group at $\mathrm{T}_{5}$ and $\mathrm{T}_{6}$. There was no statistically differences in BCS score between the three groups at $\mathrm{T} 1$ and $\mathrm{T}_{2}(P>0.05)$. No statistical differences in the incidence of adverse reactions among all groups were observed. Conclusion - Ropivacaine combined with ketolorac transverse abdominisplane block reduced the intraoperative opioid consumption, improved postoperative comfort score and do not increased the incidence of adverse reactions in gynecological laparoscopic surgery.
\end{abstract}

Keywords: ketorolac, transverse abdominis plane block, ropivacaine, gynecological laparoscopic surgery, postoperative rehabilitation

\section{Background}

Compared with open surgery, laparoscopic surgery has been widely used in gynecological surgery because of the advantages of small incision, short operation time, rapid wound healing and short hospital stay ${ }^{[1]}$. Although the incidence of postoperative discomfort in laparoscopic patients is lower than that in conventional gynecological surgery, $35-80 \%$ of laparoscopic patients still suffer from postoperative pain, which lasts up to 72 hours $^{[2]}$. Therefore, effective analgesic methods are needed to reduce discomfort after gynecologic laparoscopic surgery.

In recent years, TAP block have been increasingly used for postoperative analgesia in abdominal surgery due to the widespread use of ultrasound-guided nerve block techniques, which have greatly improved the success rate and safety. Ketorolac is a non-steroidal anti-inflammatory drug (NSAID) with strong analgesic and anti-inflammatory efficacy and is indicated for preoperative analgesia ${ }^{[3,4]}$. These two analgesic methods has become an important component of multimodal analgesia. In this study, we observed the effects of ropivacaine combined with ketorolac transverse abdominis plane block on postoperative analgesia in patients undergoing gynecological laparoscopic surgery.

\section{Material and methods}

90 ASA I or II patients, aged 18-65 years and weighting 40-80 kg, scheduled for gynecological laparoscopic surgery were recruited and divided into three groups using random number table: ropivacaine TAP block group (R group) using $0.375 \%$ ropivacaine bilateral TAP block, each side $20 \mathrm{ml}$ and $1 \mathrm{ml}$ saline intravenously; ropivacaine combined with ketorolac $15 \mathrm{mg}$ each side for TAP block group (KR group) using $0.375 \%$ ropivacaine added $15 \mathrm{mg}$ ketorolac bilateral TAP block, each side $20 \mathrm{ml}$ and $1 \mathrm{ml}$ of saline intravenously, ropivacaine for TAP block and ketorolac $30 \mathrm{mg}$ intravenously group (KIVR 
group) using $0.375 \%$ ropivacaine bilateral TAP block, $20 \mathrm{ml}$ each side and $30 \mathrm{mg}$ ketorolac intravenously, with 30 patients in each group. Patinets are excluded if they had BMI $>35 \mathrm{~kg} / \mathrm{m}^{2}$ or $<18 \mathrm{~kg} / \mathrm{m}^{2}$, had a history of severe cardiac, hepatic and renal dysfunction, psychiatric disorders and coagulation disorders; were allergy to NSAID, opioids, ropivacaine or other drugs; had used pain management drugs within 24 before surgery. The study was approved by our hospital ethics committee and all patients signed a written informed consent prior to inclusion in the study.

All patients enrolled were administrated by the same team of gynecologists and anesthesiologists. All patients fasted and abstained from food and drink for $8 \mathrm{~h}$ before surgery and were not sedated with preanesthetic drugs, and after admission to the operating room, upper limb venous access was routinely created.Electrocardiogram (ECG), heart rate (HR), oxygen saturation (SPO2), and noninvasive blood pressure (NIBP) were monitored. End-tidal carbon dioxide partial pressure (PETCO2) and bispectral index (BIS) were monitored intraoperatively. An opaque envelope was distributed by the researcher responsible for statistical analysis when entering the operating room with the patient and was opened by another anesthesiologist, which contained the grouping and instructions for the preparation of the drug solution. After sedation with $2 \mathrm{mg}$ of midazolam (Jiangsu Enhua, Liyuexi) intravenously, the patient was treated with a bilateral TAP block under ultrasound guidance by an experienced attending physician. The drug solution was configured by the anesthesiologist who opened the envelope ( $0.75 \%$ ropivacaine (AstraZeneca, Nelapin) $10 \mathrm{ml}$ with saline to $20 \mathrm{ml}$ or $15 \mathrm{mg}$ ketorolac (Sichuan Hua'ao, Shangruixin) $+0.75 \%$ ropivacaine $10 \mathrm{ml}$ with saline to $20 \mathrm{ml}$ ) and $20 \mathrm{ml}$ of the drug solution was injected on each side, and $1 \mathrm{ml}$ of the solution (30mg ketorolac or normal saline) was injected intravenously by the anesthesiologist who opened the envelope after the TAP block. The TAP block performer was unaware of the composition of the solution. General anesthesia induction was started after 15 minutes of TAP block as measured by a successful block, and those who failed the block were excluded from the group. Tracheal intubation was performed after rapid induction using sufentanil (Yichang Renfu, Renfu Shufen) 0.3-0.4ug/kg, rocuronium bromide (Zhejiang Xianju, Xianju) 0.9mg/kg, and propofol (AstraZeneca, Deprenylma) $1-2 \mathrm{mg} / \mathrm{kg}$. Anesthesia was maintained at a BIS of 40-60 and PETCO2 35-45 mmHg by administration of remifentanil $\left(0.3 \mathrm{ug} \cdot \mathrm{kg}^{-1} \cdot \mathrm{min}^{-1}\right)$ and propofol $(4-6 \mathrm{mg} / \mathrm{kg})$. 5ug sufentanil was administered intravenously when the systolic blood pressure (SAP) increased more than $20 \%$ of the basal value, while ephedrine $5 \mathrm{mg}$ was injected as the SAP decreased more than 20\%. Propofol was discontinued 10 min before the end of surgery, and remifentanil was pumped until the patient was awake and extubated. After the operation, the patient was sent to PACU for observation for $1 \mathrm{~h}$ and then sent back to the ward. $50 \mathrm{mg}$ tramadol was administered intravenously when the patient's BCS score $\leq 1$, and another 50 mg tramadol was injected if the effect was not ideal after $15 \mathrm{~min}$. If the BCS score was still $\leq 1$ after two consecutive injections, the case was excluded from the research.

General information such as patient's age, height, weight, BMI, operation time, blood loss, intraoperative sufentanil, remifentanil consumption, BCS comfort score at each time point of $2 \mathrm{~h}\left(\mathrm{~T}_{1}\right), 4 \mathrm{~h}\left(\mathrm{~T}_{2}\right), 8 \mathrm{~h}\left(\mathrm{~T}_{3}\right), 12 \mathrm{~h}\left(\mathrm{~T}_{4}\right), 16 \mathrm{~h}\left(\mathrm{~T}_{5}\right), 24 \mathrm{~h}\left(\mathrm{~T}_{6}\right)$ postoperatively were recorded. Adverse effects such as nausea, vomiting, respiratory depression, local anesthetic intoxication, and gastrointestinal bleeding were recorded.

Count data were expressed as median (interquartile spacing) (M (Q)) or percentages, using Pearson $x^{2}$ test or Fisher's exact probability method; measured data conforming to normal distribution were expressed as mean \pm standard deviation $( \pm \mathrm{s})$, using one-way ANOVA test and Bonferroni analysis for comparison between groups; non-normal distribution was expressed as median (interquartile spacing) $(\mathrm{M}(Q)$ ), using Kruskal-Wallis test.All reported $\mathrm{P}$ values are two-sided, with $\mathrm{P}$ values $<0.05$ considered statistically significant. All statistical analyses were performed using the IBM Statistical Package for the Social Sciences software for Windows, version 22.0.

\section{Results}

From December 2020 to March 2021, 90 patients were recruited. All patients were included in the study, and none met the exclusion criteria. Table 1 summarizes the baseline characteristics of the patients in the three groups. There were no significant differences among the three groups in age, BMI, ASA grades, mean arterial pressure (MAP) and heart rate (HR) when entering operation room, duration of operation and blood loss volume $(P>0.05)$. 
Table 1. Baseline characteristics of the patients ( ( $\bar{x} \pm$ s) or $M(Q))$

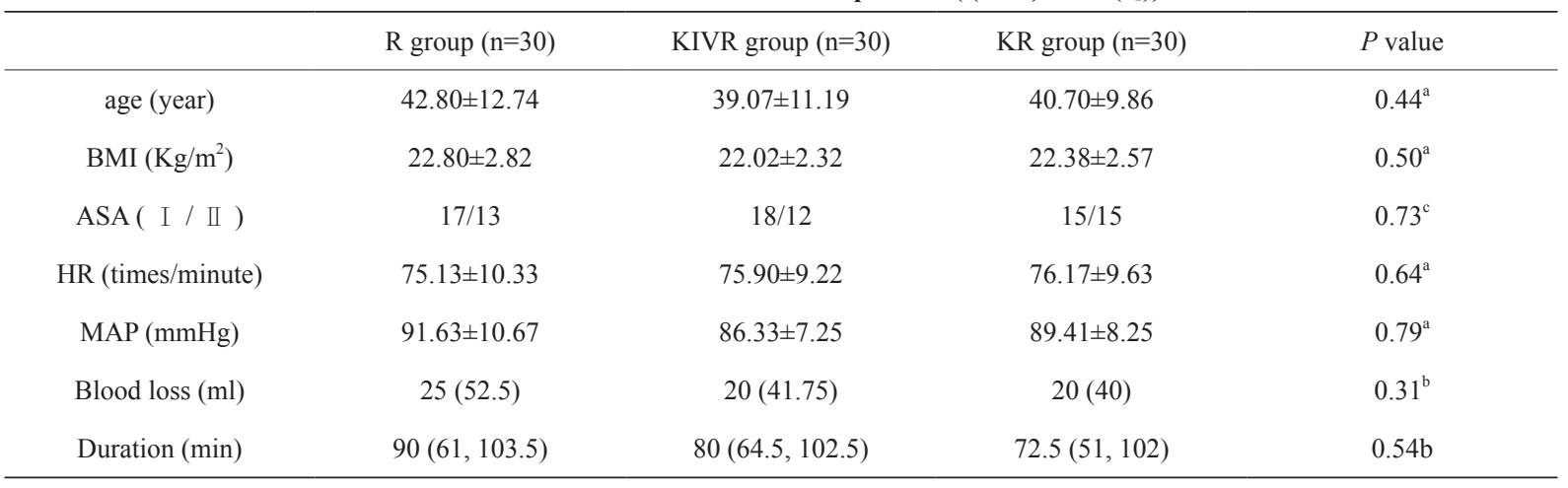

Note: ${ }^{\mathrm{a}} P>0.05$, one-way ANOVA test; ${ }^{\mathrm{b}} P>0.05$, Kruskal-Wallis test; ${ }^{\mathrm{c}} P>0.05$, Pearson $x^{2}$ test.

Table 2 shows that the total intraoperative sufentanil dosage was higher in patients in group $\mathrm{R}$ than in group KIVR and group KR in all 3 groups $(P<0.05)$; the total remifentanil dosage was higher in patients in group $\mathrm{R}$ than in group $\mathrm{KR}(P<$ 0.05 ); the difference in intraoperative sufentanil and remifentanil consumption between patients in group KIVR and group KR was not statistically significant $(P>0.05)$.

Table 2. Intraoperative opioid dosage in three groups $(\bar{x} \pm \mathrm{s})$

\begin{tabular}{cccc}
\hline & R group $(\mathrm{n}=30)$ & KIVR group $(\mathrm{n}=30)$ & KR group $(\mathrm{n}=30)$ \\
\hline sufentanil $(\mathrm{ug})$ & $30.5 \pm 5.4^{\mathrm{a}, \mathrm{b}}$ & $26.5 \pm 6.0$ & $26.0 \pm 4.8$ \\
remifentanil $(\mathrm{mg})$ & $1.1 \pm 0.4 \mathrm{~b}$ & $1.0 \pm 0.48$ & $0.8 \pm 0.3$ \\
\hline
\end{tabular}

Note: ${ }^{a} P>0.05$, compare with KIVR group, one-way ANOVA test; ${ }^{\mathrm{b}} P>0.05$, compare with KIVR group, one-way ANOVA test.

Table 3 shows that postoperative BCS scores at $\mathrm{T}_{3}, \mathrm{~T}_{4}, \mathrm{~T}_{5}$, and $\mathrm{T}_{6}$ were lower in the R group than that in the KR group in the three groups $(P<0.05)$; the $\mathrm{R}$ group was lower than the KIVR group at $\mathrm{T}_{3}$ and $\mathrm{T}_{4}(P<0.05)$, and the KIVR group was lower than the KR group at $\mathrm{T}_{5}$ and $\mathrm{T}_{6}$. There were no statistical difference in the postoperative BCS scores among the groups at $\mathrm{T}_{1}$ and $\mathrm{T}_{2}(P>0.05)$

Table 3. Postoperative BCS scores at T1-T6 in the three groups $(\mathrm{M}(Q))$

\begin{tabular}{|c|c|c|c|c|c|c|}
\hline & $\mathrm{T}_{1}$ & $\mathrm{~T}_{2}$ & $\mathrm{~T}_{3}$ & $\mathrm{~T}_{4}$ & $\mathrm{~T}_{5}$ & T6 \\
\hline $\mathrm{R}$ group $(\mathrm{n}=30)$ & $4(0)$ & $4(0)$ & $3(0.25)^{a, b}$ & $2(1)^{\mathrm{a}, \mathrm{b}}$ & $2(1)^{b}$ & $1(1)^{\mathrm{b}}$ \\
\hline KIVR group $(n=30)$ & $4(0)$ & $4(0)$ & $4(0.25)$ & $3(0)$ & $2(1)^{b}$ & $1(1)^{\mathrm{b}}$ \\
\hline KR group $(n=30)$ & $4(0)$ & $4(0)$ & $4(1)$ & $3(1)$ & $3(2)$ & $3(1)$ \\
\hline
\end{tabular}

Note: ${ }^{\mathrm{a}} P>0.05$, compare with KIVR group, Kruskal-Wallis test; ${ }^{\mathrm{b}} P>0.05$, compare with KIVR group, Kruskal-Wallis test.

The incidence of nausea and vomiting was higher in the R group (46.67\%) than in the KIVR group (33.33\%) and KR group (23.33\%), but the difference was not statistically significant $\left(x^{2}=3.9, P=0.14\right)$. No other adverse reactions such as chest distress, respiratory depression, local anesthetic intoxication, gastrointestinal bleeding and complications related to TAP block were observed in this study.

\section{Discussion}

The anterior abdominal wall consists of three layers of muscles named the external oblique abdominis, the internal oblique abdominis and the transversus abdominis. The transversus abdominis plane (TAP) lies between the internal oblique and transversus abdominis muscles and forms a gap through which the ventral branch of T7-L1 passes and where the nerves innervating these muscles are located ${ }^{[5]}$.Ultrasound-guided injection of local anesthetic solution into this location can block the anterior branch of the T7-L1 nerve and mediate intraoperative and postoperative analgesia in lower abdomen ${ }^{[6]}$.

Douglas J et al. employed local anesthetic drugs mixed with ketorolac for peripheral nerve block in podiatric surgery, demonstrating that the effect of ketorolac mixed with local anesthetic drugs for peripheral nerve block was better than that of local anesthetic drugs alone and there were no damaging effect on peripheral nerves. The physical and chemical properties of local anesthetic drugs remained relatively stable after one week of mixing with ketorolac, which proved that the solution 
of ketorolac mixed with local anesthetic drugs for peripheral nerve block was safe ${ }^{[7]}$.

The results of this study showed that ketorolac reduced intraoperative opioid dosage not only intravenously, but by TAP blockade and that both modes of administration achieved the same effect with no difference in efficacy. This result is consistent with Qi Jiang et al. ${ }^{[2]}$ The BCS scores at different time points in the three groups showed that the combined TAP block with ketorolac and ropivacaine was superior to TAP block with ropivacaine alone or intravenous injection of ketorolac combined with TAP block with ropivacaine, which is consistent with the findings of some investigators ${ }^{[2,8-10]}$, but different from the conclusions of others ${ }^{[11-13]}$. One possible reason for these results may due to the fact that the nerve block site is different.The site of block in the present study is TAP while the targets of those studies are brachial plexus nerve block or local infiltration of the surgical incision, respectively.Another reason maybe related to the different local anesthetic drugs used, both of which use lidocaine or bupivacaine while the local anesthetic used in the present study is ropivacaine.

The incidence of nausea and vomiting among the three groups showed a significantly higher incidence in group $\mathrm{R}$ than in the other two groups, which may be due to the higher intraoperative opioid dosage in group R. However, the difference was not statistically significant probably due to the sample size was not enough. There was no statistical difference in other adverse effects among the groups, which suggests that ketorolac combine with ropivacaine TAP block does not increase the incidence of adverse effects, which requires further observation.

This study is an independent, double-blind, prospective research. The TAP block operator, the researcher who opened the envelope and configured the solution and who followed up postoperatively were not involved in the design of this study. Meanwhile, the random numbers and data analysis were administrated by another two researcheres. The present study that ropivacaine combine with ketorolac TAP block contribute to multimodal analgesia and thus promote postoperative analgesia in gynecological laparoscopic patients, whick is clinically relevant and practical. The main limitation of this study is that this study is a single center study thus a multicenter large sample study is required. Another deficiency is the sample size of this study is relatively insufficient in testing the efficacy on postoperative nausea and vomiting.

\section{References}

[1] GUO S, DUANG G, WANG J, et al. Comparison of sufentanil-tramadol PCIA between laparoscopic cholecystectomy and gynecological laparoscopy [J]. Chin J Surg, 2015, 53 (02): 150-4.

[2] JIANG Q, HUANG S Q, JIAO J, et al. Effect of the Combination of Ketorolac and Bupivacaine on Transversus Abdominis Plane Block for Postoperative Analgesia After Gynecological Laparoscopic Surgery [J]. Med Sci Monit, 2020, 26 (e925006.

[3] FANG X, ZHU T, MI W, et al. Ketorolac analgesia expert consensus [J]. Journal of Clinical Anesthesiology, 2019, 35 (3): 298-303.

[4] J.M.B.SMALLMAN, H.POWELL, M.C.EWART, et al. Ketorolac for postoperative analgesia in elderly patients [J]. Anaesthesia, 2016, 149-52.

[5] MCDONNELl J G, O’DONNELl B, CURLEY G, et al. The analgesic efficacy of transversus abdominis plane block after abdominal surgery: a prospective randomized controlled trial [J]. Anesth Analg, 2007, 104 (1): 193-7.

[6] MCDONNELl J G, O’DONNELL B D, FARRELL T, et al. Transversus Abdominis Plane Block: A Cadaveric and Radiological Evaluation [J]. REGIONAL ANESTHESIA AND PAIN MEDICINE, 2007, 399-404.

[7] REINHART, DOUGLAS, J., et al. Postoperative Analgesia After Peripheral Nerve Block for Podiatric Surgery [J]. Regional Anesthesia and Pain Medicine, 2000, 25 (5): 506-13.

[8] CANSINO C, EDELMAN A, BURKE A, et al. Paracervical block with combined ketorolac and lidocaine in first-trimester surgical abortion: a randomized controlled trial [J]. Obstetrics \& Gynecology, 2009, 114 (6): 1220-6.

[9] BASENKO I L, TCHUEV P N, BUDNYUK A A, et al. Use of Ketorolac as adjuvant in brachial plexus block with bupivacaine: A-472 [J]. European Journal of Anaesthesiology, 2006, 23 (Supplement 37).

[10] BARNEY E Z, PEDRO C D, GAMEZ B H, et al. Ropivacaine and Ketorolac Wound Infusion for Post-Cesarean Delivery Analgesia: A Randomized Controlled Trial [J]. Obstetrics and Gynecology, 2020, 135 (2): 427-35.

[11] MIRKHESHTI A, SAADATNIAKI A, SALIMI A, et al. Effects of dexmedetomidine versus ketorolac as local anesthetic adjuvants on the onset and duration of infraclavicular brachial plexus block [J]. Anesth Pain Med, 2014,4 (3): e17620.

[12] CLERC S, VUILLEUMIER H, FRASCAROLO P, et al. Is the effect of inguinal field block with $0.5 \%$ bupivacaine on postoperative pain after hernia repair enhanced by addition of ketorolac or S $(+)$ ketamine? [J]. Clinical Journal of Pain, 2005, 21 (1): 101-5.

[13] BEN. B, BAUNE. U, GOLDIK. Z, et al. Is preoperative ketorolac a useful adjunct to regional anesthesia for inguinal herniorrhaphy? [J]. Acta Anaesthesiologica Scandinavica, 1996, 358-63. 